Research Article

\title{
The European Vulnerable Option Pricing with Jumps Based on a Mixed Model
}

\author{
Chao Wang, Jianmin He, and Shouwei Li \\ School of Economics and Management, Southeast University, Nanjing 211189, China \\ Correspondence should be addressed to Jianmin He; hejianminedu@163.com
}

Received 9 September 2016; Accepted 22 November 2016

Academic Editor: Chris Goodrich

Copyright (c) 2016 Chao Wang et al. This is an open access article distributed under the Creative Commons Attribution License, which permits unrestricted use, distribution, and reproduction in any medium, provided the original work is properly cited.

In this paper, we combine the reduced-form model with the structural model to discuss the European vulnerable option pricing. We define that the default occurs when the default process jumps or the corporate goes bankrupt. Assuming that the underlying asset follows the jump-diffusion process and the default follows the Vasicek model, we can have the expression of European vulnerable option. Then we use the measure transformation and martingale method to derive the explicit solution of it.

\section{Instruction}

Vulnerable option is a kind of option with credit risk. The pricing of credit risk has been concerned by scholars for a long time. Merton (1974) [1] firstly introduced option pricing into zero coupon bond with credit risk. He assumed that the capital structure of corporate consisted of two parts which are assets and liabilities; the default occurred when the corporate was insolvent at maturity. This is the basic of structural model. Black and Cox (1976) [2] researched the corporate bond pricing with subordinated debt capital structure. They improved the definition of default and took the interest and dividend into consideration to obtain the corporate bond pricing. Johnson and Stulz (1987) [3] discussed the option pricing with credit risk based on the structural model and put forward the conception of vulnerable option firstly. Longstaff and Schwartz (1995) [4] assumed that the default boundary was a constant and the recovery rate was an exogenous ratio. Then they derived the zero coupon bond pricing. Hull and White (1995) [5] assumed that the underlying asset and the counterparty asset were independent of each other and derived the vulnerable option pricing. Jarrow and Turnbull (2000) [6] presented the reduced-form model. They considered that the occurrence of default was a kind of jumps and they used the Poisson process with constant intensity to describe the density of default. Rich (1996) [7] presented that the default could occur before maturity and the equity was exercised immediately if default occurred. Klein (1996) [8] supposed that the underlying assets and credit risk were correlated and deduced the option pricing with martingale method. Madan and Unal (1998) [9] put forward a model with stochastic default intensity. According to Lando (1998) [10], the default-free bond pricing method was used for default bond pricing by adjusting the shortterm interest rate. Assuming that the default occurs at any time with specific probability, Duffie and Singleton (1999) [11] deduced the default bond pricing at initial time. Klein and Inglis (2001) [12] took the stochastic default boundary which depends on options and counterparty debts into consideration to discuss option pricing. Ammann (2002) [13] deduced the explicit solution of vulnerable option by using structural method on the assumptions of stochastic interest and boundary. The unexpected risks were introduced into default by Zhou (2001) [14]; he assumed that the corporate assets were composed of continuous process and jump process and presented the corporate assets value model based on the jump-diffusion process firstly. Hui et al. (2003) [15] discussed the pricing model with dynamic default boundary and derived the explicit solution of the vulnerable option by using the method of partial differential equation. Lakner and Liang (2008) [16] studied the credit default bonds pricing based on the structure model and the reduced-form 
model by using martingale method. Wang and Wang (2010) [17] assumed that the underlying asset followed the jumpdiffusion process and derived the expression of the European vulnerable option pricing under the Markov regime switching model. Tchuindjo (2011) [18] studied the pricing of bond and bond option under the condition of stochastic default intensity and obtained the explicit solution. Su and Wang (2012) [19] assumed that the default intensity followed the stochastic model with jumps; then the vulnerable option pricing was given based on the reduced-form model by the martingale method. Wang et al. (2015) [20] deduced the explicit solution of the European vulnerable option which was derived by the fractional Brownian motion with jumps. Yoon and Kim (2015) [21] used double Mellin transforms to study European vulnerable options under constant as well as stochastic interest rates and obtained an analytic closed-form pricing formula in each interest rate case. Fard (2015) [22] obtained a closed-form price for the vulnerable option by using the Esscher transform under a completely random generalized jump-diffusion model. Wang (2016) [23] presented a pricing model which allows for the correlation between the intensity of default and the variance of the underlying asset and derived a closed-form solution for the vulnerable option. Lee et al. (2016) [24] studied the pricing of European-type vulnerable options when the underlying asset follows the Heston dynamics and obtained a closedform analytic formula of the option price as a stochastic volatility extension of the classical Heston formula. Jeon et al. (2017) [25] studied the pricing of vulnerable path-dependent options using double Mellin transforms and obtained an explicit form pricing formula or semianalytic formula in each path-dependent option.

\section{The European Vulnerable Option Pricing Model}

Suppose that the uncertainty in the economy is described by the probability space $\left(\Omega, \mathscr{F}, Q,\left(\mathscr{F}_{t}\right)_{0 \leq t \leq T}\right)$ where $Q$ is a risk neutral martingale measure in which the discounted assets price is a martingale. The underlying assets and corporate assets are given by

$$
\begin{aligned}
& d S_{t}=(r-\theta \bar{\lambda}) S_{t} d t+\sigma_{1} S_{t} d W_{1}(t)+S_{t} d J(t), \\
& d V_{t}=r V_{t} d t+\sigma_{2} V_{t} d W_{2}(t)
\end{aligned}
$$

where $\sigma_{1}, \sigma_{2}$, and $r$ are constants and $W_{1}(t)$ and $W_{2}(t)$ are Brownian motions in the probability space. $J(t)=\sum_{i=1}^{N_{t}} Y_{i}$ is a composite Poisson process and $Y_{i}$ (set $Y_{0}=0$ ) is the jump range of it. $Y_{i}$ is a sequence of independent identically distributed random variables with the finite expected value and $E\left[Y_{i}\right]=\theta \cdot Y_{i}, W_{1}(t), W_{2}(t), W_{3}(t)$, and $N_{t}$ are independent of each other where $N_{t}$ is a Poisson process with parameter $\bar{\lambda}$. Suppose that $Y_{i}+1$ follows log-normal distribution, then we have $\ln \left(Y_{i}+1\right) \sim N\left(\mu_{J}, \sigma_{J}^{2}\right)$.
Suppose that default intensity $\lambda(t)$ is $\mathscr{F}_{t}$-measurable in the space and it follows the Vasicek model in the risk neutral measure

$$
d \lambda(t)=\alpha[\beta-\lambda(t)] d t+\sigma_{3} d W_{3}(t),
$$

where $\alpha, \beta$, and $\sigma_{3}$ are all constants. The covariance matrix of $W_{1}(t), W_{2}(t)$, and $W_{3}(t)$ is

$$
\left(\begin{array}{ccc}
1 & \rho_{12} & \rho_{13} \\
\rho_{12} & 1 & \rho_{23} \\
\rho_{13} & \rho_{23} & 1
\end{array}\right) t .
$$

We will combine the reduced-form model with the structural model to discuss the European vulnerable option pricing. We define that the default occurs when the default process jumps or the corporate goes bankrupt. Suppose that the underlying asset follows the jump-diffusion process and the corporate asset follows the Brownian motion. Then we can have the European vulnerable option pricing at initial time in the risk neutral measure. We assume that the maturity is $T$, the strike price is $K$, the default time is $\tau$, the proportion of bankruptcy costs in writer's assets is $w$, and the default boundary is a constant $D$; then the European vulnerable option pricing is

$$
\begin{aligned}
C & (0, T)=E\left[e^{-\int_{0}^{T} r(u) d u}\left(S_{T}-K\right)^{+}\right. \\
\cdot & \left.\left(\frac{1-w}{D} V_{T}\left(1-I_{\left\{\tau>T, V_{T}>D\right\}}\right)+I_{\left\{\tau>T, V_{T}>D\right\}}\right) \mid \mathscr{F}_{0}\right] \\
& =E\left[e^{-\int_{0}^{T} r(u) d u} \frac{1-w}{D} V_{T}\left(S_{T}-K\right)^{+} \mid \mathscr{F}_{0}\right] \\
& +E\left[e^{-\int_{0}^{T} r(u) d u}\left(S_{T}-K\right)^{+} I_{\left\{\tau>T, V_{T}>D\right\}} \mid \mathscr{F}_{0}\right] \\
& -E\left[e^{-\int_{0}^{T} r(u) d u} \frac{1-w}{D} V_{T}\left(S_{T}-K\right)^{+} I_{\left\{\tau>T, V_{T}>D\right\}} \mid \mathscr{F}_{0}\right] .
\end{aligned}
$$

Since $\mathscr{F}_{0}$ is a filter space and $T \geq 0$, we have $\mathscr{F}_{0} \subseteq \mathscr{F}_{T}$. Suppose that there is no default at initial time. According to the law of iterated expectations and Fubini theorem we have

$$
\begin{aligned}
E\left[I_{\{\tau>T\}} \mid \mathscr{F}_{0}\right] & =E\left[E\left[I_{\{\tau>T\}} \mid \mathscr{F}_{0}\right] \mid \mathscr{F}_{T}\right] \\
& =E\left[E\left[I_{\{\tau>T\}} \mid \mathscr{F}_{T}\right] \mid \mathscr{F}_{0}\right] .
\end{aligned}
$$

Since the path of $\{\lambda(t): 0 \leq t \leq T\}$ is known at time $T$, then

$$
\begin{aligned}
E\left[I_{\{\tau>T\}} \mid \mathscr{F}_{T}\right] & =P(\tau>T)=P\left(N_{T}-N_{0}=0\right) \\
& =\exp \left(-\int_{0}^{T} \lambda(u) d u\right) .
\end{aligned}
$$

So we have

$$
\begin{gathered}
C(0, T)=E\left[e^{-\int_{0}^{T} r(u) d u} \frac{1-w}{D} V_{T}\left(S_{T}-K\right)^{+} \mid \mathscr{F}_{0}\right] \\
+E\left[e^{-\int_{0}^{T}[r(u)+\lambda(u)] d u}\left(S_{T}-K\right)^{+} I_{\left\{V_{T}>D\right\}} \mid \mathscr{F}_{0}\right]
\end{gathered}
$$




$$
\begin{aligned}
& -E\left[e^{-\int_{0}^{T}[r(u)+\lambda(u)] d u} \frac{1-w}{D} V_{T}\left(S_{T}-K\right)^{+}\right. \\
& \left.\cdot I_{\left\{V_{T}>D\right\}} \mid \mathscr{F}_{0}\right] .
\end{aligned}
$$

\section{The Explicit Solution of European Vulnerable Option Pricing}

Suppose that the assets price follows the jump-diffusion process, then the measure transformation of the continuous diffusion process can be derived by Girsanov's theorem and the measure transformation of the jump-diffusion process is given in Theorem 1.

Theorem 1. In the probability space $(\Omega, \mathscr{F}, Q)$, if one has Radon-Nikodym derivative $Z(t)$

$$
Z(t) \mid \mathscr{F}_{t}=e^{\sum_{i=0}^{N_{t}} \ln \left(Y_{i}+1\right)-\theta \lambda t},
$$

where $\{N(t), t \geq 0\}$ is a Poisson process with intensity $\lambda$ and $Y_{i}$ is a sequence of independent identically distributed random variables that the mean is $\theta$ and $\ln \left(Y_{i}+1\right) \sim N\left(\mu_{J}, \sigma_{J}^{2}\right)$, then $N(t)$ can be transformed into measure $\widetilde{Q}$ by $Z(t)$ where the intensity $\widetilde{\lambda}=(1+\theta) \lambda$.

Proof. According to the definition of moment generating function, we have

$$
\begin{aligned}
\widetilde{E} & {\left[e^{u \sum_{i=0}^{N_{t}} \ln \left(Y_{i}+1\right)} \mid \mathscr{F}_{t}\right]=E\left[e^{u \sum_{i=0}^{N_{t}} \ln \left(Y_{i}+1\right)} Z(t) \mid \mathscr{F}_{t}\right] } \\
& =E\left[e^{(u+1) \sum_{i=0}^{N_{t}} \ln \left(Y_{i}+1\right)-\theta \lambda t} \mid \mathscr{F}_{t}\right] \\
& =\exp \left\{\lambda t\left(\varphi_{J_{(u+1)}}-1\right)-\theta \lambda t\right\} \\
& =\exp \left\{\lambda t E\left[e^{(u+1) \ln \left(Y_{i}+1\right)}\right]-\lambda t-\theta \lambda t\right\} \\
& =\exp \left\{\lambda t E\left[e^{(u+1) \ln \left(Y_{i}+1\right)}-(1+\theta)\right]\right\} \\
& =\exp \left\{\lambda t(1+\theta) E\left[\frac{e^{(u+1) \ln \left(Y_{i}+1\right)}}{1+\theta}-1\right]\right\} .
\end{aligned}
$$

$$
\begin{aligned}
& \widetilde{\bar{\lambda}}=(1+\theta) \bar{\lambda}, \\
& \rho=\frac{\rho_{12} \sigma_{1} \sigma_{2} T}{\sqrt{\sigma_{1}^{2} T+\sigma_{2}^{2} T}},
\end{aligned}
$$$$
M(u, T, \alpha)=\frac{1}{\alpha}\left(1-e^{-\alpha(T-u)}\right),
$$$$
A(0, T)=\exp \left\{-\beta T-[\lambda(0)-\beta] M(0, T, \alpha)+\frac{1}{2} \sigma_{3}^{2} \int_{0}^{T} M^{2}(u, T, \alpha) d u\right\},
$$$$
X(0, T)=S_{0} \exp \left\{r T-\rho_{13} \sigma_{1} \sigma_{3} \int_{0}^{T} M(u, T, \alpha) d u\right\},
$$

$$
E\left[e^{\ln \left(Y_{i}+1\right)}\right]=1+\theta
$$

we have

$$
\begin{aligned}
\widetilde{E} & {\left[e^{u \sum_{i=0}^{N_{t}} \ln \left(Y_{i}+1\right)} \mid \mathscr{F}_{t}\right] } \\
& =\exp \left\{\lambda t(1+\theta) E\left[e^{u \ln \left(Y_{i}+1\right)}-1\right]\right\} .
\end{aligned}
$$

Let $g=\ln \left(Y_{i}+1\right)$; then

$$
\begin{aligned}
\widetilde{E} & {\left[e^{u \sum_{i=0}^{N_{t}} \ln \left(Y_{i}+1\right)} \mid \mathscr{F}_{t}\right] } \\
& =\exp \left\{\tilde{\lambda} t\left[\int_{-\infty}^{\infty} e^{u g} f(g) d g-1\right]\right\} .
\end{aligned}
$$

So in condition $\mathscr{F}_{t}$, the intensity of $N(t)$ is $\tilde{\lambda}=(1+\theta) \lambda$.

Theorem 2. The European vulnerable option pricing at initial time in the risk neutral measure is

$$
\begin{aligned}
& C(0, T)=\sum_{n=0}^{\infty} \frac{(\tilde{\bar{\lambda}} T)^{n}}{n !} e^{-\tilde{\bar{\lambda}} T}\left[\frac{1-w}{D}\right. \\
& \cdot S_{0} V_{0} e^{r T+\rho_{12} \sigma_{1} \sigma_{2} T} N\left(d_{1}(n)\right)+e^{-r T} A(0, T) X(0, T) \\
& \cdot N\left(d_{3}(n), d_{4}(n), \rho\right)-e^{-r T} \frac{1-w}{D} A(0, T) \\
& \left.\cdot Z(0, T) N\left(d_{7}(n), d_{8}(n), \rho\right)\right] \\
& -\sum_{n=0}^{\infty} \frac{(\bar{\lambda} T)^{n}}{n !} e^{-\bar{\lambda} T}\left[\frac{1-w}{D} K V_{0} N\left(d_{2}(n)\right)\right. \\
& +e^{-r T} K A(0, T) N\left(d_{5}(n), d_{6}(n), \rho\right)-e^{-r T} \frac{1-w}{D} \\
& \left.\cdot K A(0, T) Y(0, T) N\left(d_{9}(n), d_{10}(n), \rho\right)\right],
\end{aligned}
$$

where 


$$
\begin{aligned}
& Y(0, T)=V_{0} \exp \left\{r T-\rho_{23} \sigma_{2} \sigma_{3} \int_{0}^{T} M(u, T, \alpha) d u\right\}, \\
& Z(0, T)=S_{0} V_{0} \exp \left\{2 r T-\left(\rho_{13} \sigma_{1}+\rho_{23} \sigma_{2}\right) \sigma_{3} \int_{0}^{T} M(u, T, \alpha) d u+\rho_{12} \sigma_{1} \sigma_{2}\right\}, \\
& d_{1}(n)=\frac{\ln \left(S_{0} / K\right)+r T+(1 / 2) \sigma_{1}^{2} T+\rho_{12} \sigma_{1} \sigma_{2} T-\theta \tilde{\bar{\lambda}} T+n \mu_{J}}{\sqrt{\sigma_{1}^{2} T+n \sigma_{J}^{2}}}, \\
& d_{2}(n)=\frac{\ln \left(S_{0} / K\right)+r T-(1 / 2) \sigma_{1}^{2} T+\rho_{12} \sigma_{1} \sigma_{2} T-\theta \bar{\lambda} T+n \mu_{J}}{\sqrt{\sigma_{1}^{2} T+n \sigma_{J}^{2}}}, \\
& d_{3}(n)=\frac{\ln \left(S_{0} / K\right)+r T+(1 / 2) \sigma_{1}^{2} T-\rho_{13} \sigma_{1} \sigma_{3} \int_{0}^{T} M(u, T, \alpha) d u-\theta \tilde{\bar{\lambda}} T+n \mu_{J}}{\sqrt{\sigma_{1}^{2} T+n \sigma_{J}^{2}}} \\
& d_{4}(n)=\frac{\ln \left(V_{0} / D\right)+r T-(1 / 2) \sigma_{2}^{2} T+\rho_{12} \sigma_{1} \sigma_{2} T-\rho_{23} \sigma_{2} \sigma_{3} \int_{0}^{T} M(u, T, \alpha) d u}{\sqrt{\sigma_{2}^{2} T}} \\
& d_{5}(n)=\frac{\ln \left(S_{0} / K\right)+r T-(1 / 2) \sigma_{1}^{2} T-\rho_{13} \sigma_{1} \sigma_{3} \int_{0}^{T} M(u, T, \alpha) d u-\theta \bar{\lambda} T+n \mu_{J}}{\sqrt{\sigma_{1}^{2} T+n \sigma_{J}^{2}}}, \\
& d_{6}(n)=\frac{\ln \left(V_{0} / D\right)+r T-(1 / 2) \sigma_{2}^{2} T-\rho_{23} \sigma_{2} \sigma_{3} \int_{0}^{T} M(u, T, \alpha) d u}{\sqrt{\sigma_{2}^{2} T}}, \\
& d_{7}(n)=\frac{\ln \left(S_{0} / K\right)+r T+(1 / 2) \sigma_{1}^{2} T-\rho_{13} \sigma_{1} \sigma_{3} \int_{0}^{T} M(u, T, \alpha) d u+\rho_{12} \sigma_{1} \sigma_{2} T-\theta \tilde{\bar{\lambda}} T+n \mu_{J}}{\sqrt{\sigma_{1}^{2} T+n \sigma_{J}^{2}}}, \\
& d_{8}(n)=\frac{\ln \left(V_{0} / D\right)+r T+(1 / 2) \sigma_{2}^{2} T+\rho_{12} \sigma_{1} \sigma_{2} T-\rho_{23} \sigma_{2} \sigma_{3} \int_{0}^{T} M(u, T, \alpha) d u}{\sqrt{\sigma_{2}^{2} T}}=d_{4}(n)+\sqrt{\sigma_{2}^{2} T}, \\
& d_{9}(n)=\frac{\ln \left(S_{0} / K\right)+r T-(1 / 2) \sigma_{1}^{2} T-\rho_{13} \sigma_{1} \sigma_{3} \int_{0}^{T} M(u, T, \alpha) d u+\rho_{12} \sigma_{1} \sigma_{2} T-\theta \bar{\lambda} T+n \mu_{J}}{\sqrt{\sigma_{1}^{2} T+n \sigma_{J}^{2}}} \\
& d_{10}(n)=\frac{\ln \left(V_{0} / D\right)+r T+(1 / 2) \sigma_{2}^{2} T-\rho_{23} \sigma_{2} \sigma_{3} \int_{0}^{T} M(u, T, \alpha) d u}{\sqrt{\sigma_{2}^{2} T}}=d_{6}(n)+\sqrt{\sigma_{2}^{2} T} .
\end{aligned}
$$

Proof. For convenience, we define $C(0, T)=I+I I-I I I$, where

$$
\begin{aligned}
& I=E\left[e^{-r T} \frac{1-w}{D} V_{T}\left(S_{T}-K\right)^{+} \mid \mathscr{F}_{0}\right], \\
& I I=E\left[e^{-r T-\int_{0}^{T} \lambda(u) d u}\left(S_{T}-K\right)^{+} I_{\left\{V_{T}>D\right\}} \mid \mathscr{F}_{0}\right], \\
& I I I \\
& =E\left[e^{-r T-\int_{0}^{T} \lambda(u) d u} \frac{1-w}{D} V_{T}\left(S_{T}-K\right)^{+} I_{\left\{V_{T}>D\right\}} \mid \mathscr{F}_{0}\right] .
\end{aligned}
$$

Then

$$
\begin{aligned}
I= & \frac{1-w}{D} S_{0} V_{0} e^{r T+\rho_{12} \sigma_{1} \sigma_{2} T} \sum_{n=0}^{\infty} \frac{(\tilde{\bar{\lambda}} T)^{n}}{n !} e^{-\overline{\bar{\lambda}} T} N\left(d_{1}(n)\right) \\
& -\frac{1-w}{D} K V_{0} \sum_{n=0}^{\infty} \frac{(\bar{\lambda} T)^{n}}{n !} e^{-\bar{\lambda} T} N\left(d_{2}(n)\right), \\
I I & =e^{-r T} A(0, T) X(0, T)
\end{aligned}
$$




$$
\begin{aligned}
& \sum_{n=0}^{\infty} \frac{(\tilde{\bar{\lambda}} T)^{n}}{n !} e^{-\overline{\bar{\lambda}} T} N\left(d_{3}(n), d_{4}(n), \rho\right) \\
& -e^{-r T} K A(0, T) \\
& \cdot \sum_{n=0}^{\infty} \frac{(\bar{\lambda} T)^{n}}{n !} e^{-\bar{\lambda} T} N\left(d_{5}(n), d_{6}(n), \rho\right), \\
I I I & =-e^{-r r} \frac{1-w}{D} A(0, T) Z(0, T) \\
& \cdot \sum_{n=0}^{\infty} \frac{(\overline{\bar{\lambda}} T)^{n}}{n !} e^{-\tilde{\bar{\lambda}} T} N\left(d_{7}(n), d_{8}(n), \rho\right)-e^{-r T} \frac{1-w}{D} \\
& \cdot K A(0, T) Y(0, T) \\
& \cdot \sum_{n=0}^{\infty} \frac{(\bar{\lambda} T)^{n}}{n !} e^{-\bar{\lambda} T} N\left(d_{9}(n), d_{10}(n), \rho\right) .
\end{aligned}
$$

The derivation of $I, I I$, and $I I I$ is separately shown in Proof $I$, Proof $I I$, and Proof $I I I$.

Proof $I$. We define $I=E\left[e^{-r T}((1-w) / D) V_{T}\left(S_{T}-K\right)^{+} \mid \mathscr{F}_{0}\right]=$ $I_{1}-I_{2}$, where

$$
\begin{aligned}
& I_{1}=E\left[e^{-r T} \frac{1-w}{D} S_{T} V_{T} I_{\left\{S_{T}>K\right\}} \mid \mathscr{F}_{0}\right], \\
& I_{2}=E\left[e^{-r T} \frac{1-w}{D} K V_{T} I_{\left\{S_{T}>K\right\}} \mid \mathscr{F}_{0}\right] .
\end{aligned}
$$

We deduce $I_{2}$ firstly. We introduce a new measure

$$
\begin{aligned}
\frac{d Q^{V}}{d Q} \mid \mathscr{F}_{0} & =\frac{V_{T}}{E\left[V_{T} \mid \mathscr{F}_{0}\right]} \\
& =\exp \left\{\int_{0}^{T} \sigma_{2} d W_{2}(u)-\frac{1}{2} \int_{0}^{T} \sigma_{2}^{2} d u\right\}
\end{aligned}
$$

According to (2)

$$
V_{T}=V_{0} \exp \left\{r T-\frac{1}{2} \sigma_{2}^{2} T+\int_{0}^{T} \sigma_{2} d W_{2}(u)\right\}
$$

then

$$
\begin{gathered}
I_{2}=\frac{1-w}{D} K V_{0} E\left[\exp \left\{\int_{0}^{T} \sigma_{2} d W_{2}(u)-\frac{1}{2} \sigma_{2}^{2} T\right\}\right. \\
\left.\cdot I_{\left\{S_{T}>K\right\}} \mid \mathscr{F}_{0}\right]=\frac{1-w}{D} K V_{0} E^{V}\left[I_{\left\{S_{T}>K\right\}} \mid \mathscr{F}_{0}\right] .
\end{gathered}
$$

Using (19) and Girsanov's theorem, we have

$$
\begin{aligned}
& W_{1}^{V}(T)=W_{1}(T)-\rho_{12} \sigma_{2} T, \\
& W_{2}^{V}(T)=W_{2}(T)-\sigma_{2} T .
\end{aligned}
$$

According to (1)

$$
\begin{aligned}
S_{T} & =S_{0} \exp \left\{r T-\frac{1}{2} \sigma_{1}^{2} T\right. \\
& \left.+\int_{0}^{T} \sigma_{1} d W_{1}(u)-\theta \bar{\lambda} T+\sum_{i=0}^{N_{T}} \ln \left(Y_{i}+1\right)\right\} .
\end{aligned}
$$

So in the measure $Q^{V}$, we have

$$
\begin{aligned}
S_{T} & =S_{0} \exp \left\{r T-\frac{1}{2} \sigma_{1}^{2} T+\int_{0}^{T} \sigma_{1} d W_{1}^{V}(u)\right. \\
& \left.+\rho_{12} \sigma_{1} \sigma_{2} T-\theta \bar{\lambda} T+\sum_{i=0}^{N_{T}} \ln \left(Y_{i}+1\right)\right\} .
\end{aligned}
$$

Since

$$
\int_{0}^{T} \sigma_{1} d W_{1}^{V}(u) \sim N\left(0, \sigma_{1}^{2} T\right)
$$

and when $N_{T}=n$,

$$
\sum_{i=0}^{N_{t}} \ln \left(Y_{i}+1\right) \sim N\left(n \mu_{J}, n \sigma_{J}^{2}\right),
$$

then we have

$$
E^{V}\left[I_{\left\{S_{T}>K\right\}} \mid \mathscr{F}_{0}\right]=\sum_{n=0}^{\infty} \frac{(\bar{\lambda} T)^{n}}{n !} e^{-\bar{\lambda} T} N\left(d_{2}(n)\right),
$$

where

$$
\begin{aligned}
& d_{2}(n) \\
& =\frac{\ln \left(S_{0} / K\right)+r T-(1 / 2) \sigma_{1}^{2} T+\rho_{12} \sigma_{1} \sigma_{2} T-\theta \bar{\lambda} T+n \mu_{J}}{\sqrt{\sigma_{1}^{2} T+n \sigma_{J}^{2}}} .
\end{aligned}
$$

Then we will deduce $I_{1}$. We introduce a new measure

$$
\begin{aligned}
& \frac{d Q^{S V}}{d Q} \mid \mathscr{F}_{0}=\frac{S_{T} V_{T}}{E\left[S_{T} V_{T} \mid \mathscr{F}_{0}\right]}=\exp \left\{\int_{0}^{T} \sigma_{1} d W_{1}(u)\right. \\
& +\int_{0}^{T} \sigma_{2} d W_{2}(u)-\frac{1}{2}\left(\sigma_{1}^{2}+\sigma_{2}^{2}+2 \rho_{12} \sigma_{1} \sigma_{2}\right) T \\
& \left.-\theta \bar{\lambda} T+\sum_{i=0}^{N_{T}} \ln \left(Y_{i}+1\right)\right\} .
\end{aligned}
$$

According to Girsanov's theorem and Theorem 1

$$
W_{1}^{S V}(T)=W_{1}(T)-\sigma_{1} T-\rho_{12} \sigma_{2} T
$$


So in measure $Q^{S V}$

$$
\begin{aligned}
S_{T} & =S_{0} \exp \left\{r T+\frac{1}{2} \sigma_{1}^{2} T+\int_{0}^{T} \sigma_{1} d W_{1}^{S V}(u)\right. \\
& \left.+\rho_{12} \sigma_{1} \sigma_{2} T-\theta \tilde{\bar{\lambda}} T+\sum_{i=0}^{N_{T}} \ln \left(Y_{i}+1\right)\right\} .
\end{aligned}
$$

Since

$$
E\left[S_{T} V_{T} \mid \mathscr{F}_{0}\right]=S_{0} V_{0} e^{2 r T+\rho_{12} \sigma_{1} \sigma_{2} T}
$$

we have

$$
I_{1}=\frac{1-w}{D} S_{0} V_{0} e^{r T+\rho_{12} \sigma_{1} \sigma_{2} T} E^{S V}\left[I_{\left\{S_{T}>K\right\}} \mid \mathscr{F}_{0}\right]
$$

When $N_{T}=n$

$$
E^{S V}\left[I_{\left\{S_{T}>K\right\}} \mid \mathscr{F}_{0}\right]=\sum_{n=0}^{\infty} \frac{(\tilde{\bar{\lambda}} T)^{n}}{n !} e^{-\tilde{\bar{\lambda}} T} N\left(d_{1}(n)\right),
$$

where

$$
\begin{aligned}
& d_{1}(n) \\
& =\frac{\ln \left(S_{0} / K\right)+r T+(1 / 2) \sigma_{1}^{2} T+\rho_{12} \sigma_{1} \sigma_{2} T-\theta \tilde{\bar{\lambda}} T+n \mu_{J}}{\sqrt{\sigma_{1}^{2} T+n \sigma_{J}^{2}}} .
\end{aligned}
$$

Proof II. We define II $=E\left[e^{-r T-\int_{0}^{T} \lambda(u) d u}\left(S_{T}-K\right)^{+} I_{\left\{V_{T}>D\right\}} \mid\right.$ $\left.\mathscr{F}_{0}\right]=I_{3}-I_{4}$, where

$$
\begin{aligned}
& I_{3}=E\left[e^{-r T-\int_{0}^{T} \lambda(u) d u} S_{T} I_{\left\{S_{T}>K, V_{T}>D\right\}} \mid \mathscr{F}_{0}\right], \\
& I_{4}=E\left[e^{-r T-\int_{0}^{T} \lambda(u) d u} K I_{\left\{S_{T}>K, V_{T}>D\right\}} \mid \mathscr{F}_{0}\right] .
\end{aligned}
$$

We deduce $I_{4}$ firstly. We define an equivalent martingale measure $Q^{\lambda}$ according to Radon-Nikodym derivative as follows:

$$
\frac{d Q^{\lambda}}{d Q} \mid \mathscr{F}_{0}=\frac{e^{-\int_{0}^{T} \lambda(u) d u}}{E\left[e^{-\int_{0}^{T} \lambda(u) d u} \mid \mathscr{F}_{0}\right]}
$$

According to (3)

$$
\begin{aligned}
-\int_{0}^{T} \lambda(u) d u= & -\beta T-[\lambda(0)-\beta] M(0, T, \alpha) \\
& -\sigma_{3} \int_{0}^{T} M(u, T, \alpha) d W_{3}(u) .
\end{aligned}
$$
have

Let $A(0, T)=E\left[e^{-\int_{0}^{T} \lambda(u) d u} \mid \mathscr{F}_{0}\right]$; using Itô lemma we

$$
\begin{aligned}
& A(0, T)=\exp \{-\beta T-[\lambda(0)-\beta] M(0, T, \alpha) \\
& \left.\quad+\frac{1}{2} \sigma_{3}^{2} \int_{0}^{T} M^{2}(u, T, \alpha) d u\right\} .
\end{aligned}
$$

So

$$
\begin{aligned}
& \frac{d Q^{\lambda}}{d Q} \mid \mathscr{F}_{0}=\frac{e^{-\int_{0}^{T} \lambda(u) d u}}{E\left[e^{-\int_{0}^{T} \lambda(u) d u} \mid \mathscr{F}_{0}\right]} \\
& \quad=\exp \left\{-\sigma_{3} \int_{0}^{T} M(u, T, \alpha) d W_{3}(u)\right. \\
& \left.-\frac{1}{2} \sigma_{3}^{2} \int_{0}^{T} M^{2}(u, T, \alpha) d u\right\}
\end{aligned}
$$

Using Girsanov’s theorem,

$$
\begin{aligned}
& W_{1}^{\lambda}(T)=W_{1}(T)+\rho_{13} \sigma_{3} \int_{0}^{T} M(u, T, \alpha) d u, \\
& W_{2}^{\lambda}(T)=W_{2}(T)+\rho_{23} \sigma_{3} \int_{0}^{T} M(u, T, \alpha) d u \\
& W_{3}^{\lambda}(T)=W_{3}(T)+\sigma_{3} \int_{0}^{T} M(u, T, \alpha) d u .
\end{aligned}
$$

Then the solutions of $S_{T}$ and $V_{T}$ in measure $Q^{\lambda}$ are

$$
\begin{aligned}
S_{T} & =S_{0} \exp \left\{r T-\frac{1}{2} \sigma_{1}^{2} T\right. \\
& \left.+\int_{0}^{T} \sigma_{1} d W_{1}(u)-\theta \bar{\lambda} T+\sum_{i=0}^{N_{T}} \ln \left(Y_{i}+1\right)\right\}=S_{0} \\
& \cdot \exp \left\{r T-\frac{1}{2} \sigma_{1}^{2} T+\int_{0}^{T} \sigma_{1} d W_{1}^{\lambda}(u)\right. \\
& -\rho_{13} \sigma_{1} \sigma_{3} \int_{0}^{T} M(u, T, \alpha) d u-\theta \bar{\lambda} T \\
& \left.+\sum_{i=0}^{N_{T}} \ln \left(Y_{i}+1\right)\right\}, \\
V_{T} & =V_{0} \exp \left\{r T-\frac{1}{2} \sigma_{2}^{2} T+\int_{0}^{T} \sigma_{2} d W_{2}(u)\right\}=V_{0} \\
& \cdot \exp \left\{r T-\frac{1}{2} \sigma_{2}^{2} T+\int_{0}^{T} \sigma_{2} d W_{2}^{\lambda}(u)\right. \\
& \left.-\rho_{23} \sigma_{2} \sigma_{3} \int_{0}^{T} M(u, T, \alpha) d u\right\} \cdot
\end{aligned}
$$


Since

$$
\begin{aligned}
I_{4} & =E\left[e^{-r T-\int_{0}^{T} \lambda(u) d u} K I_{\left\{S_{T}>K, V_{T}>D\right\}} \mid \mathscr{F}_{0}\right] \\
& =e^{-r T} K E\left[e^{-\int_{0}^{T} \lambda(u) d u} I_{\left\{S_{T}>K, V_{T}>D\right\}} \mid \mathscr{F}_{0}\right] \\
& =e^{-r T} K A(0, T) E^{\lambda}\left[I_{\left\{S_{T}>K, V_{T}>D\right\}} \mid \mathscr{F}_{0}\right],
\end{aligned}
$$

$$
\begin{aligned}
& d_{5}(n)=\frac{\ln \left(S_{0} / K\right)+r T-(1 / 2) \sigma_{1}^{2} T-\rho_{13} \sigma_{1} \sigma_{3} \int_{0}^{T} M(u, T, \alpha) d u-\theta \bar{\lambda} T+n \mu_{J}}{\sqrt{\sigma_{1}^{2} T+n \sigma_{J}^{2}}}, \\
& d_{6}(n)=\frac{\ln \left(V_{0} / D\right)+r T-(1 / 2) \sigma_{2}^{2} T-\rho_{23} \sigma_{2} \sigma_{3} \int_{0}^{T} M(u, T, \alpha) d u}{\sqrt{\sigma_{2}^{2} T}} .
\end{aligned}
$$

Then we will deduce $I_{3}$. We define a new measure

$$
\frac{d Q^{S}}{d Q^{\lambda}} \mid \mathscr{F}_{0}=\frac{S_{T}}{E^{\lambda}\left[S_{T} \mid \mathscr{F}_{0}\right]} .
$$

Let $X(0, T)=E^{\lambda}\left[S_{T} \mid \mathscr{F}_{0}\right]$; according to (42)

$$
X(0, T)=S_{0} \exp \left\{r T-\rho_{13} \sigma_{1} \sigma_{3} \int_{0}^{T} M(u, T, \alpha) d u\right\}
$$

Then

$$
\begin{aligned}
& \frac{d Q^{S}}{d Q^{\lambda}} \mid \mathscr{F}_{0}=\exp \left\{-\frac{1}{2} \sigma_{1}^{2} T+\int_{0}^{T} \sigma_{1} d W_{1}^{\lambda}(u)-\theta \bar{\lambda} T\right. \\
& \left.\quad+\sum_{i=0}^{N_{T}} \ln \left(Y_{i}+1\right)\right\} .
\end{aligned}
$$

According to Girsanov's theorem and Theorem 1

$$
\begin{aligned}
& W_{1}^{S}(T)=W_{1}^{\lambda}(T)-\sigma_{1} T, \\
& W_{2}^{S}(T)=W_{2}^{\lambda}(T)-\rho_{12} \sigma_{1} T .
\end{aligned}
$$

when $N_{T}=n$, substituting $S_{T}$ and $V_{T}$ into $I_{\left\{S_{T}>K, V_{T}>D\right\}}$, we have

$$
\begin{aligned}
E^{\lambda} & {\left[I_{\left\{S_{T}>K, V_{T}>D\right\}} \mid \mathscr{F}_{0}\right] } \\
& =\sum_{n=0}^{\infty} \frac{(\bar{\lambda} T)^{n}}{n !} e^{-\bar{\lambda} T} N\left(d_{5}(n), d_{6}(n), \rho\right),
\end{aligned}
$$

where

$$
\begin{aligned}
S_{T} & =S_{0} \exp \left\{r T+\frac{1}{2} \sigma_{1}^{2} T+\int_{0}^{T} \sigma_{1} d W_{1}^{S}(u)\right. \\
& -\rho_{13} \sigma_{1} \sigma_{3} \int_{0}^{T} M(u, T, \alpha) d u-\theta \widetilde{\bar{\lambda}} T \\
& \left.+\sum_{i=0}^{N_{T}} \ln \left(Y_{i}+1\right)\right\}, \\
V_{T} & =V_{0} \exp \left\{r T-\frac{1}{2} \sigma_{2}^{2} T+\int_{0}^{T} \sigma_{2} d W_{2}^{S}(u)+\rho_{12} \sigma_{1} \sigma_{2} T\right. \\
& \left.-\rho_{23} \sigma_{2} \sigma_{3} \int_{0}^{T} M(u, T, \alpha) d u\right\} .
\end{aligned}
$$

Since

$$
\begin{aligned}
I_{3} & =E\left[e^{-r T-\int_{0}^{T} \lambda(u) d u} S_{T} I_{\left\{S_{T}>K, V_{T}>D\right\}} \mid \mathscr{F}_{0}\right] \\
& =e^{-r T} E\left[e^{-\int_{0}^{T} \lambda(u) d u} S_{T} I_{\left\{S_{T}>K, V_{T}>D\right\}} \mid \mathscr{F}_{0}\right] \\
& =e^{-r T} A(0, T) E^{\lambda}\left[S_{T} I_{\left\{S_{T}>K, V_{T}>D\right\}} \mid \mathscr{F}_{0}\right] \\
& =e^{-r T} A(0, T) X(0, T) E^{S}\left[I_{\left\{S_{T}>K, V_{T}>D\right\}} \mid \mathscr{F}_{0}\right],
\end{aligned}
$$

when $N_{0}=n$,

$$
\begin{aligned}
E^{S} & {\left[I_{\left\{S_{T}>K, V_{T}>D\right\}} \mid \mathscr{F}_{0}\right] } \\
& =\sum_{n=0}^{\infty} \frac{(\tilde{\bar{\lambda}} T)^{n}}{n !} e^{-\tilde{\bar{\lambda}} T} N\left(d_{3}(n), d_{4}(n), \rho\right),
\end{aligned}
$$


where

$$
\begin{aligned}
& d_{3}(n)=\frac{\ln \left(S_{0} / K\right)+r T+(1 / 2) \sigma_{1}^{2} T-\rho_{13} \sigma_{1} \sigma_{3} \int_{0}^{T} M(u, T, \alpha) d u-\theta \tilde{\bar{\lambda}} T+n \mu_{J}}{\sqrt{\sigma_{1}^{2} T+n \sigma_{J}^{2}}}, \\
& d_{4}(n)=\frac{\ln \left(V_{0} / D\right)+r T-(1 / 2) \sigma_{2}^{2} T+\rho_{12} \sigma_{1} \sigma_{2} T-\rho_{23} \sigma_{2} \sigma_{3} \int_{0}^{T} M(u, T, \alpha) d u}{\sqrt{\sigma_{2}^{2} T}} .
\end{aligned}
$$

Proof III. We define III $=E\left[e^{-r T-\int_{0}^{T} \lambda(u) d u}((1-w) / D) V_{T}\left(S_{T}-\right.\right.$ $\left.K)^{+} I_{\left\{V_{T}>D\right\}} \mid \mathscr{F}_{0}\right]=I_{5}-I_{6}$, where

$$
\begin{aligned}
& I_{5}=E\left[e^{-r T-\int_{0}^{T} \lambda(u) d u} \frac{1-w}{D} S_{T} V_{T} I_{\left\{S_{T}>K, V_{T}>D\right\}} \mid \mathscr{F}_{0}\right], \\
& I_{6}=E\left[e^{-r T-\int_{0}^{T} \lambda(u) d u} \frac{1-w}{D} K V_{T} I_{\left\{S_{T}>K, V_{T}>D\right\}} \mid \mathscr{F}_{0}\right] .
\end{aligned}
$$

We deduce $I_{6}$ firstly. We introduce a new measure

$$
\frac{d Q^{V}}{d Q^{\lambda}} \mid \mathscr{F}_{0}=\frac{V_{T}}{E^{\lambda}\left[V_{T} \mid \mathscr{F}_{0}\right]} .
$$

Let $Y(0, T)=E^{\lambda}\left[V_{T} \mid \mathscr{F}_{0}\right]$; according to (43)

$$
Y(0, T)=V_{0} \exp \left\{r T-\rho_{23} \sigma_{2} \sigma_{3} \int_{0}^{T} M(u, T, \alpha) d u\right\} .
$$

So

$$
\frac{d Q^{V}}{d Q^{\lambda}} \mid \mathscr{F}_{0}=\exp \left\{-\frac{1}{2} \sigma_{2}^{2} T+\int_{0}^{T} \sigma_{2} d W_{2}^{\lambda}(u)\right\} .
$$

According to Girsanov's theorem,

$$
\begin{aligned}
& W_{1}^{V}(T)=W_{1}^{\lambda}(T)-\rho_{12} \sigma_{2} T, \\
& W_{2}^{V}(T)=W_{2}^{\lambda}(T)-\sigma_{2} T .
\end{aligned}
$$

So, in measure $Q^{V}$, we have

$$
S_{T}=S_{0} \exp \left\{r T-\frac{1}{2} \sigma_{1}^{2} T+\int_{0}^{T} \sigma_{1} d W_{1}^{V}(u)\right.
$$

$$
\begin{aligned}
& -\rho_{13} \sigma_{1} \sigma_{3} \int_{0}^{T} M(u, T, \alpha) d u+\rho_{12} \sigma_{1} \sigma_{2} T-\theta \bar{\lambda} T \\
& \left.+\sum_{i=0}^{N_{T}} \ln \left(Y_{i}+1\right)\right\}, \\
V_{T} & =V_{0} \exp \left\{r T+\frac{1}{2} \sigma_{2}^{2} T+\int_{0}^{T} \sigma_{2} d W_{2}^{V}(u)\right. \\
& \left.-\rho_{23} \sigma_{2} \sigma_{3} \int_{0}^{T} M(u, T, \alpha) d u\right\} .
\end{aligned}
$$

Since

$$
\begin{aligned}
I_{6} & =E\left[e^{-r T-\int_{0}^{T} \lambda(u) d u} \frac{1-w}{D} K V_{T} I_{\left\{S_{T}>K, V_{T}>D\right\}} \mid \mathscr{F}_{0}\right] \\
& =e^{-r T} \frac{1-w}{D} K E\left[e^{-\int_{0}^{T} \lambda(u) d u} V_{T} I_{\left\{S_{T}>K, V_{T}>D\right\}} \mid \mathscr{F}_{0}\right] \\
& =e^{-r T} \frac{1-w}{D} K A(0, T) E^{\lambda}\left[V_{T} I_{\left\{S_{T}>K, V_{T}>D\right\}} \mid \mathscr{F}_{0}\right] \\
& =e^{-r T} \frac{1-w}{D} K A(0, T) Y(0, T) \\
& \cdot E^{V}\left[I_{\left\{S_{T}>K, V_{T}>D\right\}} \mid \mathscr{F}_{0}\right],
\end{aligned}
$$

when $N_{0}=n$, we have

$$
\begin{aligned}
E^{V} & {\left[I_{\left\{S_{T}>K, V_{T}>D\right\}} \mid \mathscr{F}_{0}\right] } \\
= & \sum_{n=0}^{\infty} \frac{(\bar{\lambda} T)^{n}}{n !} e^{-\bar{\lambda} T} N\left(d_{9}(n), d_{10}(n), \rho\right),
\end{aligned}
$$

where

$$
\begin{aligned}
d_{9}(n) & =\frac{\ln \left(S_{0} / K\right)+r T-(1 / 2) \sigma_{1}^{2} T-\rho_{13} \sigma_{1} \sigma_{3} \int_{0}^{T} M(u, T, \alpha) d u+\rho_{12} \sigma_{1} \sigma_{2} T-\theta \bar{\lambda} T+n \mu_{J}}{\sqrt{\sigma_{1}^{2} T+n \sigma_{J}^{2}}}, \\
d_{10}(n) & =\frac{\ln \left(V_{0} / D\right)+r T+(1 / 2) \sigma_{2}^{2} T-\rho_{23} \sigma_{2} \sigma_{3} \int_{0}^{T} M(u, T, \alpha) d u}{\sqrt{\sigma_{2}^{2} T}}=d_{6}(n)+\sqrt{\sigma_{2}^{2} T} .
\end{aligned}
$$


Then we will deduce $I_{5}$. We introduce the measure as follows:

$$
\frac{d Q^{S V}}{d Q^{\lambda}} \mid \mathscr{F}_{0}=\frac{S_{T} V_{T}}{E^{\lambda}\left[S_{T} V_{T} \mid \mathscr{F}_{0}\right]} .
$$

Let $Z(0, T)=E^{\lambda}\left[S_{T} V_{T} \mid \mathscr{F}_{0}\right]$; according to (42) and (43)

$$
\begin{aligned}
& Z(0, T)=S_{0} V_{0} \exp \{2 r T \\
& -\left(\rho_{13} \sigma_{1}+\rho_{23} \sigma_{2}\right) \sigma_{3} \int_{0}^{T} M(u, T, \alpha) d u \\
& \left.\quad+\rho_{12} \sigma_{1} \sigma_{2}\right\} .
\end{aligned}
$$

So

$$
\begin{aligned}
& \frac{d Q^{S V}}{d Q^{\lambda}} \mid \mathscr{F}_{0}=\exp \left\{-\frac{1}{2}\left(\sigma_{1}^{2}+\sigma_{2}^{2}+\rho_{12} \sigma_{1} \sigma_{2}\right) T\right. \\
& \quad+\int_{0}^{T} \sigma_{1} d W_{1}^{\lambda}(u)+\int_{0}^{T} \sigma_{2} d W_{2}^{\lambda}(u)-\theta \bar{\lambda} T \\
& \left.+\sum_{i=0}^{N_{T}} \ln \left(Y_{i}+1\right)\right\} .
\end{aligned}
$$

According to Girsanov's theorem and Theorem 1

$$
\begin{aligned}
& W_{1}^{S V}(T)=W_{1}^{\lambda}(T)-\sigma_{1} T-\rho_{12} \sigma_{2} T \\
& W_{2}^{S V}(T)=W_{2}^{\lambda}(T)-\sigma_{2} T-\rho_{12} \sigma_{1} T
\end{aligned}
$$

So in measure $Q^{S V}$

$$
\begin{aligned}
S_{T} & =S_{0} \exp \left\{r T+\frac{1}{2} \sigma_{1}^{2} T+\int_{0}^{T} \sigma_{1} d W_{1}^{S V}(u)\right. \\
& -\rho_{13} \sigma_{1} \sigma_{3} \int_{0}^{T} M(u, T, \alpha) d u+\rho_{12} \sigma_{1} \sigma_{2} T-\theta \tilde{\bar{\lambda}} T \\
& \left.+\sum_{i=0}^{N_{T}} \ln \left(Y_{i}+1\right)\right\}, \\
V_{T} & =V_{0} \exp \left\{r T+\frac{1}{2} \sigma_{2}^{2} T+\int_{0}^{T} \sigma_{2} d W_{2}^{S V}(u)\right. \\
& \left.-\rho_{23} \sigma_{2} \sigma_{3} \int_{0}^{T} M(u, T, \alpha) d u+\rho_{12} \sigma_{1} \sigma_{2} T\right\} .
\end{aligned}
$$

Since

$$
\begin{aligned}
I_{5} & =E\left[e^{-r T-\int_{0}^{T} \lambda(u) d u} \frac{1-w}{D} S_{T} V_{T} I_{\left\{S_{T}>K, V_{T}>D\right\}} \mid \mathscr{F}_{0}\right] \\
& =e^{-r T} \frac{1-w}{D} E\left[e^{-\int_{0}^{T} \lambda(u) d u} S_{T} V_{T} I_{\left\{S_{T}>K, V_{T}>D\right\}} \mid \mathscr{F}_{0}\right] \\
& =e^{-r T} \frac{1-w}{D} A(0, T) E^{\lambda}\left[S_{T} V_{T} I_{\left\{S_{T}>K, V_{T}>D\right\}} \mid \mathscr{F}_{0}\right] \\
& =e^{-r T} \frac{1-w}{D} A(0, T) Z(0, T) \\
& \cdot E^{S V}\left[I_{\left\{S_{T}>K, V_{T}>D\right\}} \mid \mathscr{F}_{0}\right],
\end{aligned}
$$

when $N_{0}=n$, we have

$$
\begin{aligned}
E^{S V} & {\left[I_{\left\{S_{T}>K, V_{T}>D\right\}} \mid \mathscr{F}_{0}\right] } \\
= & \sum_{n=0}^{\infty} \frac{(\tilde{\bar{\lambda}} T)^{n}}{n !} e^{-\tilde{\bar{\lambda}} T} N\left(d_{7}(n), d_{8}(n), \rho\right),
\end{aligned}
$$

where

$$
\begin{aligned}
& d_{7}(n)=\frac{\ln \left(S_{0} / K\right)+r T+(1 / 2) \sigma_{1}^{2} T-\rho_{13} \sigma_{1} \sigma_{3} \int_{0}^{T} M(u, T, \alpha) d u+\rho_{12} \sigma_{1} \sigma_{2} T-\theta \tilde{\bar{\lambda}} T+n \mu_{J}}{\sqrt{\sigma_{1}^{2} T+n \sigma_{J}^{2}}}, \\
& d_{8}(n)=\frac{\ln (V(0) / D)+r T+(1 / 2) \sigma_{2}^{2} T+\rho_{12} \sigma_{1} \sigma_{2} T-\rho_{23} \sigma_{2} \sigma_{3} \int_{0}^{T} M(u, T, \alpha) d u}{\sqrt{\sigma_{2}^{2} T}}=d_{4}(n)+\sqrt{\sigma_{2}^{2} T} .
\end{aligned}
$$

\section{Numerical Experiments}

In this section, we mainly discuss the influence of different parameters on option value. The parameters are as follows:

$$
\begin{aligned}
& \alpha=0.2 ; \\
& \beta=0.05 ;
\end{aligned}
$$

$$
r(0)=0.05
$$

$$
\theta=1
$$$$
\bar{\lambda}=0.1
$$$$
\widetilde{\bar{\lambda}}=0.2
$$$$
K=100
$$ 


$$
\begin{gathered}
D=60 ; \\
T=1 ; \\
\sigma_{1}=0.5 ; \\
\sigma_{2}=0.5 ; \\
\sigma_{3}=0.5 ; \\
\sigma_{J}=0.5 ; \\
\mu_{J}=0.2 ; \\
\rho_{12}=0.5 ; \\
\rho_{23}=0.5 ; \\
\rho_{13}=0.5 ; \\
w=0.8
\end{gathered}
$$

Figure 1 shows the influences of underlying assets and corporate assets on option value. We can see that the option value increases gradually with the increase of underlying assets and corporate assets, but the corporate assets have a higher influence on option value than that of underlying assets.

From Figure 2 we can see that the jump process has an obvious influence on option pricing which can make the option value more accurate. Because of the expansion of default, the default probability becomes higher. So the option value is lower than that in Black-Scholes model and this is coincident with the reality.

Figure 3 shows the changes of option value with different jump intensity. With the increase of jump intensity, the option value will decrease because of the rising default risk. We can choose the most suitable jump intensity according to the historical data to make the option value more accurate in reality.

From Figures 4 and 5 we can see that the option value decreases obviously when the default intensity and the default boundary are less than certain critical value while the it changes slowly when the default intensity and the default boundary exceed the critical value. In real application, the default intensity and the default boundary can be set according to the corporate reputation, so that the option pricing will be more reasonable.

Figure 6 shows that the option value and the proportion of bankruptcy costs are negatively correlated. The higher the bankruptcy costs, the lower the option value.

\section{Conclusion}

The traditional definition of default probability is divided into two forms: the reduced-form model says that the corporate will default as long as the jump occurs and the default probability is determined by the default intensity while the structural model says that the default occurs when the corporate assets are less than the default boundary and the default probability is determined by the relationship

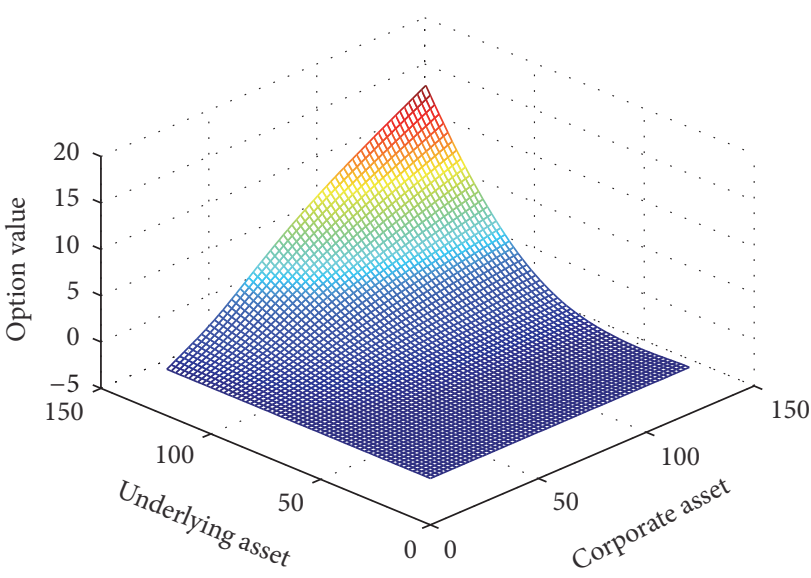

FIgURE 1: Option value with different underlying assets and corporate assets.

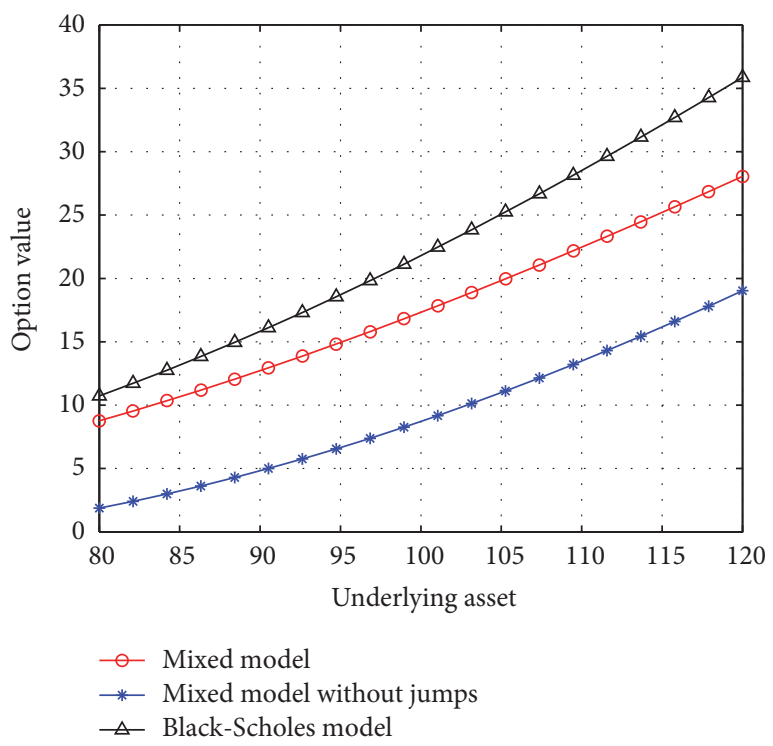

FIGURE 2: Option value in different pricing models.

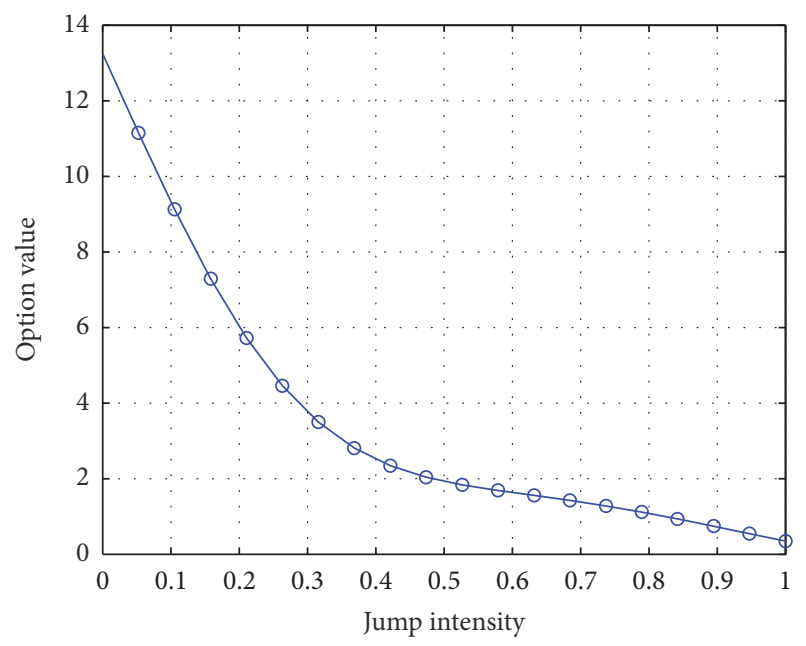

Figure 3: Option value with different jump intensity. 


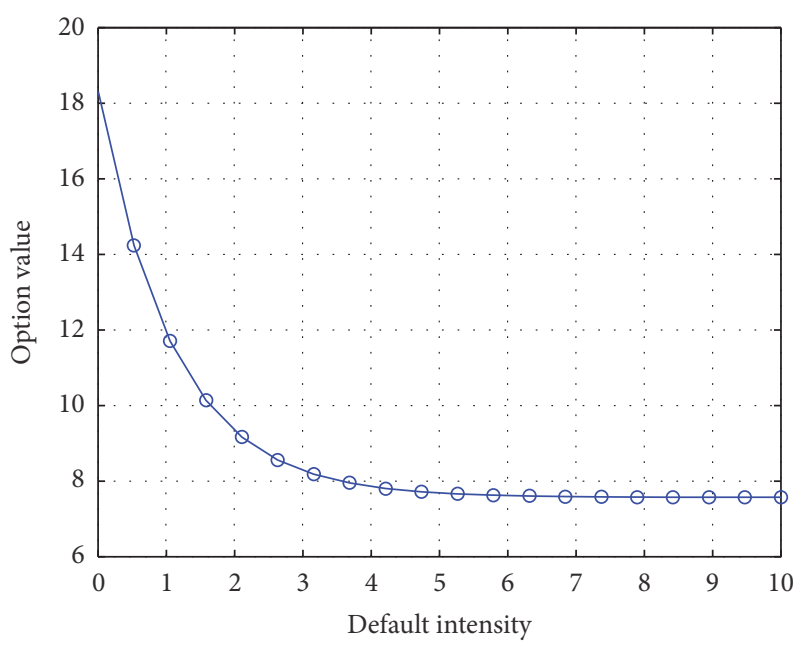

FIGURE 4: Option value with different default intensity.

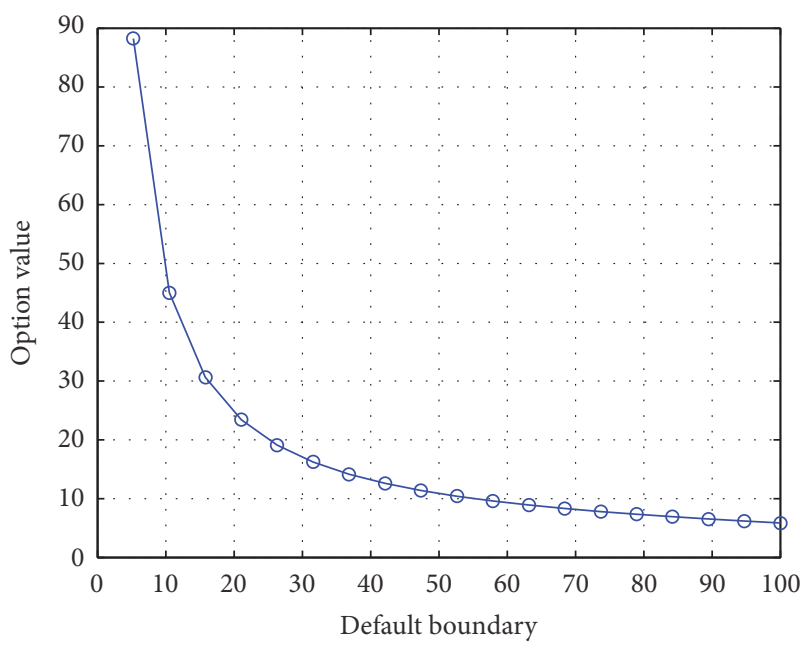

FIGURE 5: Option value with different default boundary.

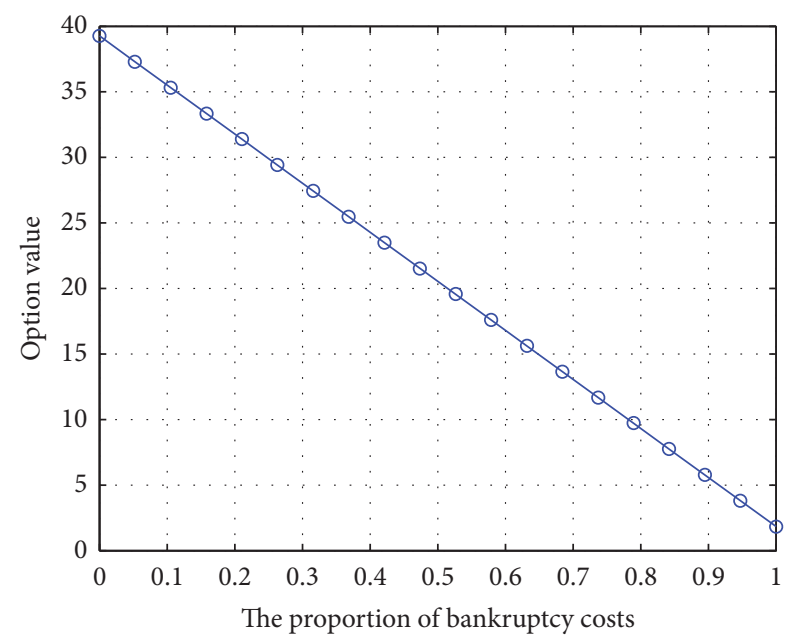

FIGURE 6: Option value with different proportion of bankruptcy costs. between corporate assets and default boundary. In this paper, we combine reduced-form model with structural model to discuss the European vulnerable option pricing. We define that the default occurs when the default process jumps or the corporate goes bankrupt. Supposing that the underlying asset follows the jump-diffusion process and the default follows the Vasicek model, we deduce the expression of vulnerable option. Then we use the measure transformation and martingale method to derive the explicit solution of it. The results show that the model we put forward is more in line with the real financial market.

\section{Competing Interests}

The authors declare that the mentioned received funding in Acknowledgments did not lead to any conflict of interests regarding the publication of this manuscript and there is no other possible conflict of interests in the manuscript.

\section{Acknowledgments}

This research is supported by NSFC (nos. 71371034; 71171051; 71201023), Teaching and Research Program for Excellent Young Teachers of Southeast University (no. 2242015R30021).

\section{References}

[1] R. C. Merton, "On the pricing of corporate debt: the risk structure of interest rates," The Journal of Finance, vol. 29, no. 2, pp. 449-470, 1974.

[2] F. Black and J. C. Cox, "Valuing corporate securities: some effects of bond indenture provisions," The Journal of Finance, vol. 31, no. 2, pp. 351-367, 1976.

[3] H. Johnson and R. Stulz, "The pricing of options with default risk," The Journal of Finance, vol. 42, no. 2, pp. 267-280, 1987.

[4] F. A. Longstaff and E. S. Schwartz, "A simple approach to valuing risky fixed and floating rate debt," The Journal of Finance, vol. 50, no. 3, pp. 789-819, 1995.

[5] J. Hull and A. White, "The impact of default risk on the prices of options and other derivative securities," Journal of Banking \& Finance, vol. 19, no. 2, pp. 299-322, 1995.

[6] R. A. Jarrow and S. M. Turnbull, "The intersection of market and credit risk," Journal of Banking \& Finance, vol. 24, no. 1-2, pp. 271-299, 2000.

[7] D. Rich, "The valuation and behavior of black-scholes options subject to intertemporal default risk," Review of Derivatives Research, vol. 1, no. 1, pp. 25-59, 1996.

[8] P. Klein, "Pricing black-scholes options with correlated credit risk," Journal of Banking \& Finance, vol. 20, no. 7, pp. 1211-1229, 1996.

[9] D. B. Madan and H. Unal, "Pricing the risks of default," Review of Derivatives Research, vol. 2, no. 2-3, pp. 121-160, 1998.

[10] D. Lando, “On cox processes and credit risky securities," Review of Derivatives Research, vol. 2, no. 2-3, pp. 99-120, 1998.

[11] D. Duffie and K. J. Singleton, "Modeling term structures of defaultable bonds," Review of Financial Studies, vol. 12, no. 4, pp. 687-720, 1999.

[12] P. Klein and M. Inglis, "Pricing vulnerable European options when the option's payoff can increase the risk of financial 
distress," Journal of Banking \& Finance, vol. 25, no. 5, pp. 9931012, 2001.

[13] M. Ammann, Credit Risk Valuation: Methods, Models and Applications, Springer Finance, Springer, Berlin, Germany, 2nd edition, 2002.

[14] C. Zhou, "The term structure of credit spreads with jump risk," Journal of Banking \& Finance, vol. 25, no. 11, pp. 2015-2040, 2001.

[15] C. H. Hui, C. Lo, and H. Lee, "Pricing Vulnerable BlackScholes Options with Dynamic Default Barriers," The Journal of Derivatives, vol. 10, no. 4, pp. 62-69, 2003.

[16] P. Lakner and W. Liang, "Optimal investment in a defaultable bond," Mathematics \& Financial Economics, vol. 1, no. 3-4, pp. 283-310, 2008.

[17] W. Wang and W. Wang, "Pricing vulnerable options under a Markov-modulated regime switching model," Communications in Statistics-Theory and Methods, vol. 39, no. 19, pp. 3421-3433, 2010.

[18] L. Tchuindjo, "Closed-form solutions for pricing credit-risky bonds and bond options," Applied Mathematics \& Computation, vol. 217, no. 13, pp. 6133-6143, 2011.

[19] X. Su and W. Wang, "Pricing options with credit risk in a reduced form model," Journal of the Korean Statistical Society, vol. 41, no. 4, pp. 437-444, 2012.

[20] C. Wang, S. Zhou, and J. Yang, "The pricing of vulnerable options in a fractional Brownian motion environment," Discrete Dynamics in Nature and Society, vol. 2015, Article ID 579213, 10 pages, 2015.

[21] J.-H. Yoon and J.-H. Kim, "The pricing of vulnerable options with double Mellin transforms," Journal of Mathematical Analysis and Applications, vol. 422, no. 2, pp. 838-857, 2015.

[22] F. A. Fard, "Analytical pricing of vulnerable options under a generalized jump-diffusion model," Insurance: Mathematics and Economics, vol. 60, pp. 19-28, 2015.

[23] X. Wang, "Analytical valuation of vulnerable options in a discrete-time framework," Probability in the Engineering and Informational Sciences, 2016.

[24] M.-K. Lee, S.-J. Yang, and J.-H. Kim, "A closed form solution for vulnerable options with Heston's stochastic volatility," Chaos, Solitons \& Fractals, vol. 86, pp. 23-27, 2016.

[25] J. Jeon, J.-H. Yoon, and M. Kang, "Pricing vulnerable pathdependent options using integral transforms," Journal of Computational and Applied Mathematics, vol. 313, pp. 259-272, 2017. 


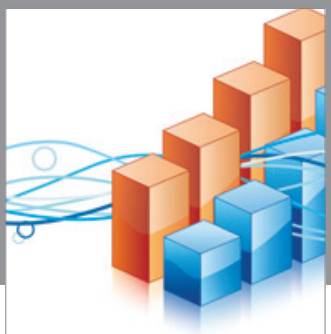

Advances in

Operations Research

vatem alat4

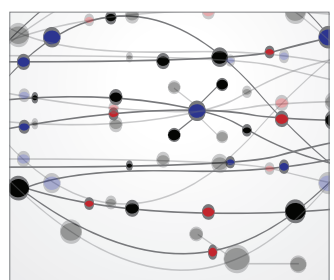

\section{The Scientific} World Journal
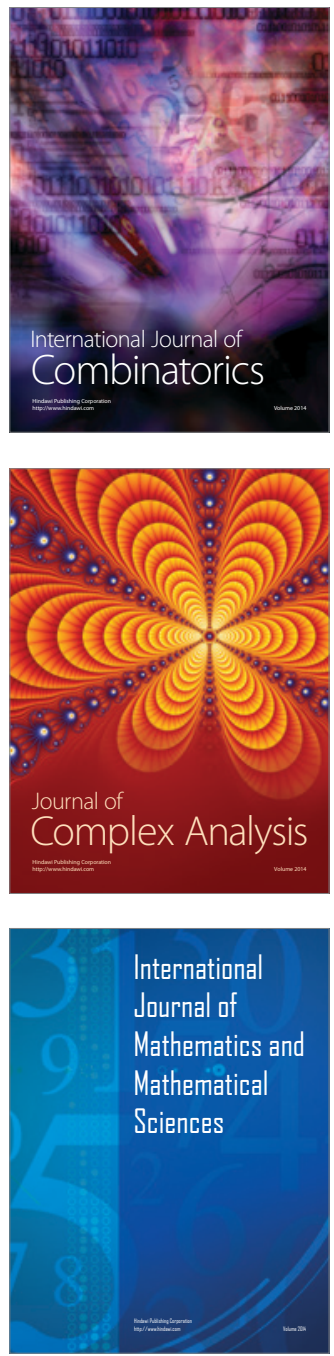
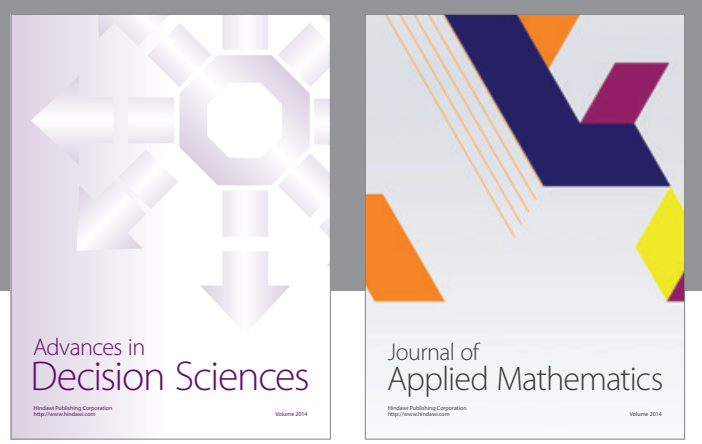

Algebra

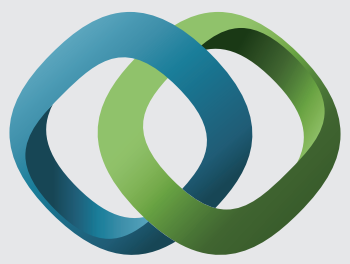

\section{Hindawi}

Submit your manuscripts at

http://www.hindawi.com
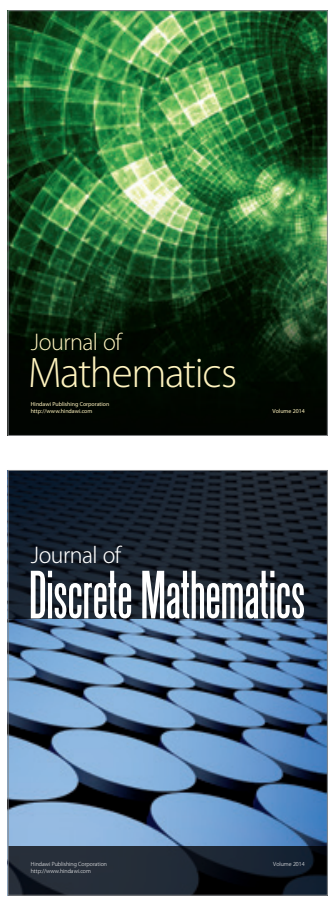

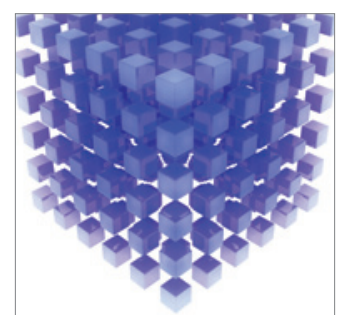

Mathematical Problems in Engineering
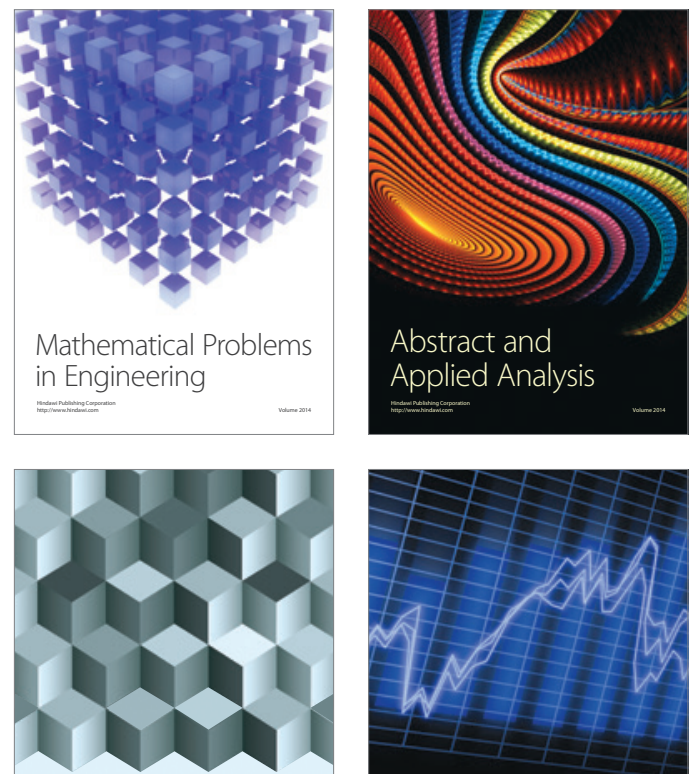

Journal of

Function Spaces

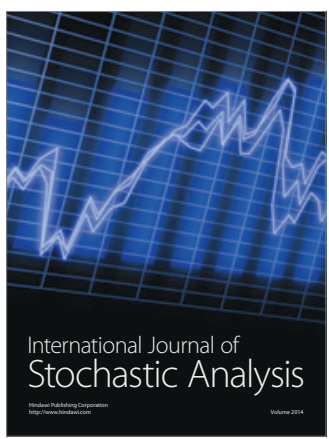

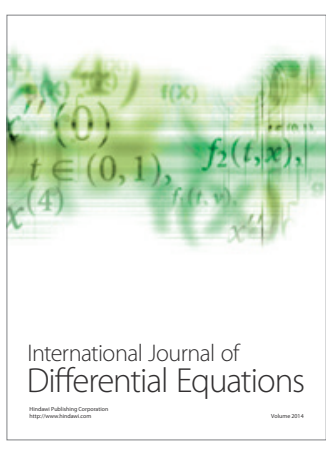
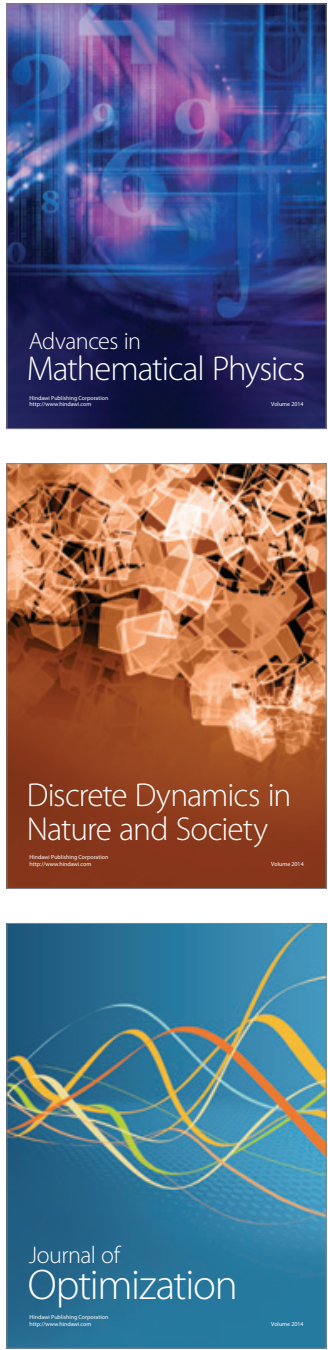